\title{
Verzeichnis der für die Literaturnachweise benutzten Abkürzungen.
}

A. = Lnarig s Annaleñ der Chemie.

A. ch. = Annales de chimie et de physique.

Am. = American Chemical Journal.

Am. Soc. $=$ Journal of the American Chemical Society.

A. Pth. = Archiv für experimentelle Pathologie und Pharmakologie.

Ar. = Archiv der Pharmazie.

B. $=$ Berichte der Deutschen Chemischen Gesellschaft.

Bio. Z. = Biochemische Zeitschrift.

Bl. = Bulletin de la Société Chimique de France.

B. Ph. P. = Beiträge zur chemischen Physiologie und Pathologie.

C. = Chemisches Zentralblatt.

C. $r$. = Comptes rendus des séances de l'Acadénie des sciences.

Ch. I. = Chemische Industrie.

Ch. Z. = Chemiker-Zeitung.

Chem. N. = Chemical News.

D. = Durarars Polytechnisches Journal. ,

D. R.P. = Patentschrift des Deutschen Reiches.

El. Ch. Z. = Elektrochemische Zeitschrift.

Fr. = (Freseniug') Zeitschrift für analytische Chemie.

Frdl. = FriedLänder s Fortschritte der Teerfarbenfabrikation (Berlin, Sprimarr).

G. = Gazzetta chimica italiana.

H. = (HOPPE-SEYLER s) Zeitschrift für physiologische Chemie.

Helv. = Helvetica Chimica Acta.

J. = Jahresbericht der Chemie.

J. pr. = Journal für praktische Chemie.

L. V. St. = Landwirtschaftliche Versuchsstationen.

M. = Monatshefte der Chemie.

'P. C. H. = Pharmazeutische Zentralhalle.

P. Ch. S. = Proceedings of the Chemical Society.

$\mathrm{Ph} . \mathrm{Ch} .=$ Zeitschrift für physikalische Chemie.

R. = Recueil des trayaux chimiques des Pays-Bas.

R. A. L. = Atti della Reale Accademia dei Lincei (Rendiconti).

Soc. $=$ Journal of the Chemical Society of London.

W. = Wiedemanns Annalen der Physik.

Z. = Zeitschrift für Chemie.

Z. a. Ch. = Zeitschrift für anorganische Chemie.

Z. Ang. = Zeitschrift für angewandte Chemie.

Z. B. = Zeitschrift für Biologie.

Z. El. Ch. = Zeitschrift für Elektrochemie.

Z. Kr. = Zeitschrift für Krystallographie. 